\title{
Requirements Scoping Visualization for Project Management
}

\author{
Krzysztof Wnuk ${ }^{1}$ and David Callele ${ }^{2}$ \\ 1 Department of Computer Science, Lund University, Lund, Sweden \\ Krzysztof.Wnuk@cs.1th.se \\ http://www.cs.lth.se \\ 2 Department of Computer Science, University of Saskatchewan, \\ Saskatchewan, Canada \\ callele@cs.usask.ca \\ http://www.cs.usask.ca/
}

\begin{abstract}
Determining requirements process efficiency, and measuring the corresponding monetary impacts, is a challenging but necessary aspect of project management. In this paper, we perfor independent analysis of scoping decisions from a large industrial oro ect yith the goal of providing visualizations that facilitate investioga ion of process efficiency, agility, and the effects of scoping dec iron. visualizations proposed in this paper can be used to ar aryz-coping dynamics and support process management decisions on antitative rather than a qualitative basis.

Keywords: Requirements visu ization, process evaluation, requirements scope, project manage
\end{abstract}

\section{Introduction}

In Market-Driven wents Engineering (MDRE) [1, the time taken to deliver the product to arket (and hence the overall release scheduling) is important and may strongy affect market success [2]. These time pressures place hard limits on all aspects of the development effort and force requirements efforts to be efficient and responsive [3]. Feature leapfrogging [4] between companies also imposes hard time constrains, which combined with resource constraints force requirements to be prioritized, with some requirements postponed for later implementation [5]. The process of selecting a subset of requirements for immediate implementation within a given project is called scoping and is considered a key activity for achieving economic benefits in product line development [6]. In MDRE, the scope of the project must adapt to competitive pressures and respond to changing market conditions in a timely manner - making appropriate scope decisions is a vital part of developing software systems that meet stakeholders' needs and expectations [5]7.

Project management in this context has the goal of delivering a quality product within the given resource constraints, with appropriate risk management and acceptable predictability. Making decisions in a timely manner is fundamental 
for productive software management - unnecessarily delaying decisions can lead to wasted resources and can place other aspects of the projects at risk due to resource constraints [8]. However, as reported by Boehm and Sullivan [9] there is a "disconnect" between the process of making technical software product decisions and the value creation criteria of the organizations that develop these products. Moreover, the growing importance of software in all aspects of successful business demands better understanding of the relationships between technical properties of the decisions and the criteria for business value creation. In other words, software development should be considered an "investment activity" that aims at maximizing value creation for the resources invested [9].

Our prior work 101112 showed that in MDRE there is the potential for the project scope to be constantly changing, or at least be under pressure to do so, particularly from unanticipated market forces. For example, the last minute addition of cutting-edge features and technologies to a project can lead to significant investments in requirements definition and feasibility analysis efforts. The associated project management risks may include excessiv resource allocations that starve other efforts, destabilizing technologies being led to the product before they are ready, and even outright project failare Ths prior work proposed new visualizations to help to assess the dyn scope changes in this context using a post-mortem analysis perspec

In this paper, we analyze scoping from a proct management perspective and propose visualizations that support managemen-decision-making. Using the action research strategy [13] the second of this paper, who is an industrial Project Manager (PM) interested in ecisfon Support Systems (DSS), reviewed the previous work with the focus financial aspects of requirements engineering and requirements scoping its implications on efficient project management. The project manag no ds tools that can help him to identify opportunities for improvement tha process itself and to reduce risk by identifying wasted resources. As a est $\$$ of this participatory effort, the following research questions are state

- What factors infuence the process of removing features from the scope of the project? (RQ1)

- What factors influence the late addition of features to the scope of the project? (RQ2)

This work investigates the acts of adding and removing features from the scope of a product release; all features under consideration for a release are present before the scoping process begins. RQ1 identifies opportunities to optimize feature removal under the premise that, if the feature is to be removed from the release, then early removal wastes fewer resources. RQ2 investigates late feature addition to identify opportunities to reduce risks inherent in this decision.

In the remainder of this paper we review the related work and present a summary of the relevant results from our prior work (Section 2). We discuss the research approach and data used in this study in Section 3. Section 4 presents new visualizations that support financial analysis of the scoping process in projects. 
We discuss threats to validity for this study in Section 5 and close with our conclusions and identification of future work in Section 6 .

\section{Related Work}

Release planning is an integral part of software product management. Multiple releases are considered in a release plan [14 and the selection of the 'right' requirements for a particular release is normally preceded by requirements prioritization [15 5] and cost estimation [16. Release planning is performed within the context of the product and corporate strategies, providing input to the feature selection process [17/1819]. The feature selection process is not a trivial task; dependencies on other features 20 or frequent changes to the scope of the next release of a project [12 are typical complications. As a result, the selection process often becomes an uneasy compromise where the development efforts toward some features may need to be sacrificed (at the expense of wasted effort) in support of other features whose priorities have changed.

Managing this scope complexity is considered one th core functions of software release planning and a key activity for achis ec nomic benefits in product line development [6]. While the importang sping has already been reported in several studies, most of the research is beu ed the domain scoping aspect and the process of scope identification 6/21]. When looking at product portfolio scoping [6] most techniques focys on tne financial benefits associated with reuse across the product line 2

The quantity of information that me be managed for large software projects is often overwhelming and visualization be useful in this context, offering more dimensions to represent than text 23 Appropriate visualizations can assist with, for example, the requiremsn conprehension problem of gaining a quick assessment of the state of a set a qu thements; a task typically impeded by the need to browse through disjoin tex yal requirements documentation and accompanying models [24]. The polizons presented in our previous work [101112] give a support assessment the scoping process for large projects and can also be used for more in-depth andysis of the details of the scoping process. In [10] the Feature Survival Chart was introduced and applied to one large industrial project. In [12] the Feature Survival Chart was complemented with a set of scope tracking measurements while in [1] the Feature Transition Chart technique, designed to cover scope changes across the projects, was proposed and initially validated. These visualizations can be extended via metrics such as the volatility of the feature set and temporal measurements such as the time taken to cancel a feature [12. In this paper, we extend the previous work, presenting a new set of visualizations that aim at supporting project managers in understanding the scoping dynamics and assess the monetary impact of the visualized scope changes.

\section{Research Approach and Data in Context}

The analysis performed in this study is based on empirical data from a large company that develops embedded systems for a global market. The company uses 
Table 1. The Project Timeline

\begin{tabular}{ll}
\hline Milestones & Project Analyzed \\
Elapsed Days & Launched May 2007 \\
\hline MS1 & 0 \\
MS2 & 98 \\
MS3 & 143 \\
MS4 & 203 \\
\hline
\end{tabular}

Table 2. Milestone Criteria

MS1 Potential features are drawn from the long-term roadmap documents. The initial scope (set of features) is defined and baselined. The scope is then documented and updated after the weekly meeting of the Change Control Board (CCB). The CCB is responsible for adding or removing features from the project plan.

MS2 Features are refined to requirements that are specified, r riewed and approved. Each feature typically contains ten or more requirementom various areas in the products. The feature requirements are forwa ed o th design teams who return updated effort estimates.

MS3 Requirements are updated as necessary (bas on design team feedback) and the effort estimates are refined.

MS4 The requirements work and design are finded. The final project scope has been negotiated with the developm resources and the project is ready to start implementation.

the software product line aper based upon a common code base (referred to here as the platform) he product line. A platform project follows the stage-gate model [26] wit sural increments; Milestones (MSs) and Tollgates (TGs) are used to con rol she project progress. In particular, there are four milestones for requ re. nus management and design before the implementation starts: MS1 through IS4. The scope of a given project is based on a unit called a feature, a group of requirements that constitute a functional enhancement to the platform. At the beginning of a project, the feature definition typically contains a functional description and estimates of market value and development effort. To cancel or descope a feature in this context means to permanently remove the feature from the project plan. The discussions in this paper focus on activity within a current project, features re-introduced in a later project are outside of the scope of this work.

The elapsed time information for the projects used in this work are presented in Table 1, The milestone criteria are presented in Table 2, We use the same metrics as in our prior work [12, summarized in Table 3 for convenience.

In this paper we have focused on the top four reasons for feature removal within the data set. The categories are defined as follows.

- Stakeholder A stakeholder made a business decision to to add or cancel the feature 
Table 3. Metrics

M1 Number of positive and negative scope changes per time stamp/baseline. A positive scope change means a feature was added, and a negative scope change indicates a feature was removed. M1 is not used in the current work and is only included here for completeness.

M2 Time to feature removal. The time from feature introduction until permanent removal.

M3 Number of state changes per feature. Number of times that the state of the feature in the scope was changed. In this work, we do not consider the initial inclusion to the scope as a scope change.

M4 Time to feature addition. The time from the start of the project until the feature was added.

M5 Reason for feature removal. A categorical metric focusing on reasons for removal due to project constraints.

- Resources A feature has been removed due to lack of rasources

- Portfolio A feature has been removed due to changes in the product line portfolio

- Replaced A feature has been replaced by an anotheature.

The underlying data sets have been transform acessary, for the purposes of this paper to ensure that they are logically oct and consistent across the projects. The analyzed project has 223 considered during the analysis period. The project contained features nus survived from project inception, features that were added during the project, and features that were canceled during the project.

For investigations involvin - nly features that were removed from the scope were included (120 d a oints). The values for M3 and M4 were calculated for all features in the an project; features that survived the cancellation process were assigned ale of zero to remove them from consideration.

For investigation ving M5, 120 descoping decisions were analyzed and categorized. The en re set of 120 cancellation decisions was used during the M2-M5 and M4-M5 correlation analyses. However, some categories such as "dependent on supplier" or "inadequate feature description" contained such a small number of data points that we focused our analyses on the five categories with the most data points. Moreover, the normalization of the dataset (for example to MS4) further limited the datasets. The specific number of data points for each category is contained in the axis labels (e.g. ( $7 \mathrm{dp})$ means 7 data points) for Figures 1, 2, 3 and 4,

\section{Visualizations}

The following sub-sections present visualizations designed to assist a project manager in analyzing the requirements scoping process. The visualizations portray the relationships between the (normalized) metric data. 
The underlying business decisions that generate the data sets are assumed to be rational in context - the decisions may not be perfect but the decisions were acceptable in the situation. Under this assumption, if a feature is going to be canceled, it is less wasteful of resources to cancel the feature sooner than later. While there may be cases where efforts to promote early feature cancellation may impede innovation, in this product line process the innovation decisions are made prior to the studied scenarios and are based on an ROI analysis rather than technical feasibility. This is not greenfield product development and innovation is incremental and often small in scope (e.g. a new software feature as compared to a new hardware platform).

\subsection{Interpreting the Visualizations}

Box-plots are a non-parametric means for visualizing datasets; they provide greater insight into the data than a simple average value without making any assumptions about the distribution of the underlying data - a factor that makes them particularly useful for smaller data sets.

The box plots used in this work have horizontal bars kers) that identify the most extreme data points for the population (ar rox imely $+/-2.7 \sigma$ and $99.3 \%$ coverage if the data are normally distrib at not considered outliers; outliers are individually plotted. The b ares the $25 \%$ to $75 \%$ range (second and third quartiles) of the data set an thedian value is represented by the horizontal line within the body of box.

In Figure 1] comparing the plot for the dataset to the other plots for subsets of the dataset, we note that he plot for the Resources category of M5 is similar in size and shape to tha entire dataset. This shape correlation identifies resource managemep principle contributor to the long-tail of the entire dataset. Five pses for the tail are identified in Section 4.2. that may or may not be the critical factor is that the visuation helps the Project Mana their attention to cancel decisions for $\mathrm{t}$ is project. In comparison, the time to add issues in Figure 3 are attributable to the stakeholders rather than resources - again directing the Project Manager's attention in an appropriate manner.

\subsection{The Relationship between the Time to Feature Removal (M2) and the Reason for Feature Removal (M5) - Addressing Research Question RQ1}

The data set was partitioned based on the Reason for Descope of the feature and the resulting temporal distributions (normalized to MS4) of the top four categories for M5 are presented as box-whisker plots in Figure 1. We see that approximately $40 \%$ of the features (29/72) were removed as a result of a stakeholder business decision with the mean time to feature removal (as a result of a stakeholder business decision) approximately $10 \%$ of the way into the final milestone of the requirements management process. These characteristics indicate that the feature list was pruned relatively aggressively and relatively quickly. 


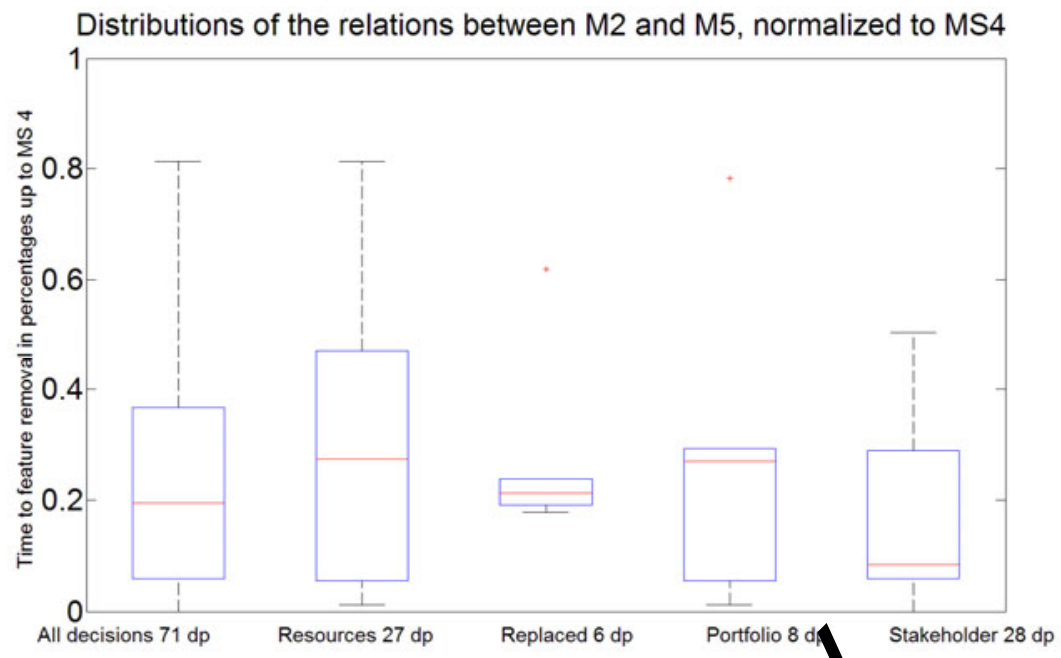

Fig. 1. The Relationship between the time to feature rem aryz and the reason for feature removal (M5), normalized to MS4

In contrast, approximately $50 \%$ of the featu median line of the Resources box in the plot) were removed from scopdue to lack of resources by approximately $30 \%$ of the way to MS4. Howejer, so features were not removed until almost the end of the project. This is matter for concern to a project manager for it may be evidence that one or pre of the following statements are true (this list is only exemplary thaustive):

- There are difficulties e .ming the scope (effort) required for a feature.

- There are difficulties d te-nining what resources are available.

- There are diffiralties mating the contributions of the available resources.

- There are diffry matching the available resources to the features (resource misma ch).

- The business process (algorithm) used to make the decisions may need improvement.

Simplistically, from the project manager's perspective, all features that are canceled waste scarce resources. It is imperative to make the keep/cancel decision for a feature as soon as possible to minimize this waste. In this regard, the keep/cancel decision could be considered a form of potential defect detection in the sense that a canceled feature is a feature that is no longer deemed appropriate for this feature release. As such, the economic benefits are those identified by Boehm and Basili [27].

Next, the earliest stage of the project was investigated, the time between MS1 and MS2 to see if we could gain any further insight. The data set was partitioned again and only features that have been removed in the time between MS1 and MS2 were kept. The resulting data was normalized to MS2 and the results are shown in Figure 2 . 


\section{Distributions of the relations between M2 and M5 NORMALIZED TO MS 2}

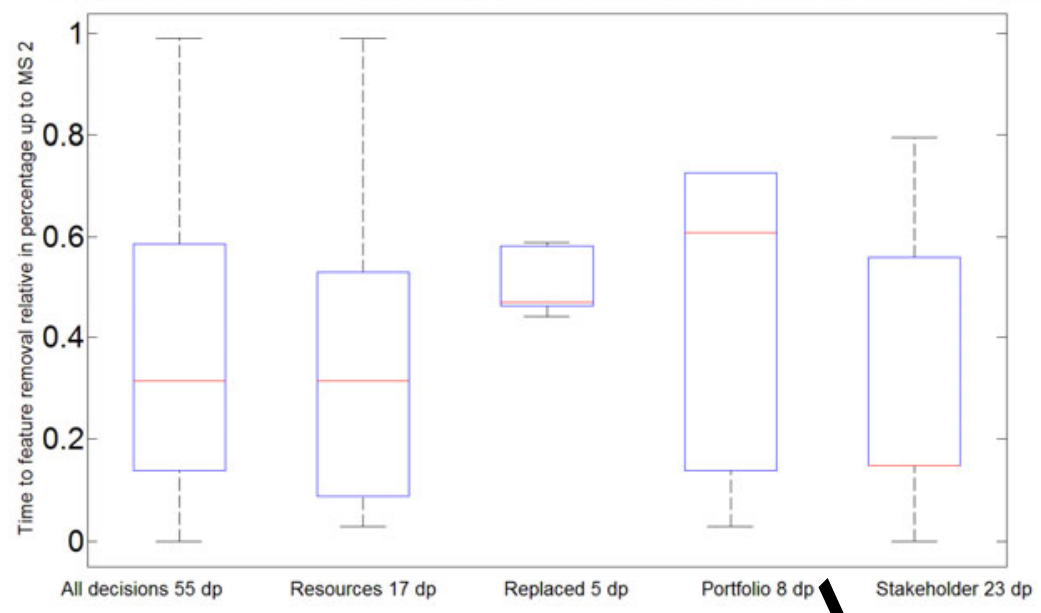

Fig. 2. The Relationship between the time to feature rem rar $\mathrm{M}$ and the reason for feature removal (M5), normalized to MS2

The resulting data represents approximate $76 \%$ of the entire data set. We note that all portfolio changes $(8 / 8)$, almest alreplaced or renamed decisions $(5 / 6)$ and most of the stakeholder bu necisions $(23 / 29)$ were made in this interval. This indicates that the proct stope is being reduced in an effective manner, although portfolio chang reduced or renamed decisions still lag the start of the interval by an avag value of approximately $50 \%$.

Only 17 of 27 resource disios were made in this interval, again with an average delay in excess of 50 the interval and a tail that extends to the end of the interval. While the s yation is not as challenging as it appeared in Figure [1. there does appe bidence that improvements could be made.

\subsection{The Relationship between the Time to Feature Addition} (M4) and the Reason for Feature Removal (M5) - Addressing Rresearch Question RQ2

Figure 3 illustrates the relationship between M4 and M5 for the analyzed project, normalized to MS4 for this project. We note that the medians for each partition show significantly greater diversity than those of Figure 1. The average time to feature introduction was approximately $50 \%$ of the timeline. We note that features introduced in the first half of the projects were predominantly cancelled due to lack of resources, replaced or renamed, or portfolio changes. Features introduced after the midpoint were predominantly cancelled as a result of stakeholder business decisons, although there is a much lesser contribution from a lack of resources.

Figure 4 illustrates the relationship between M4 and M5 normalized to MS3 for this project. Again, we note that there is significant diversity in the median 


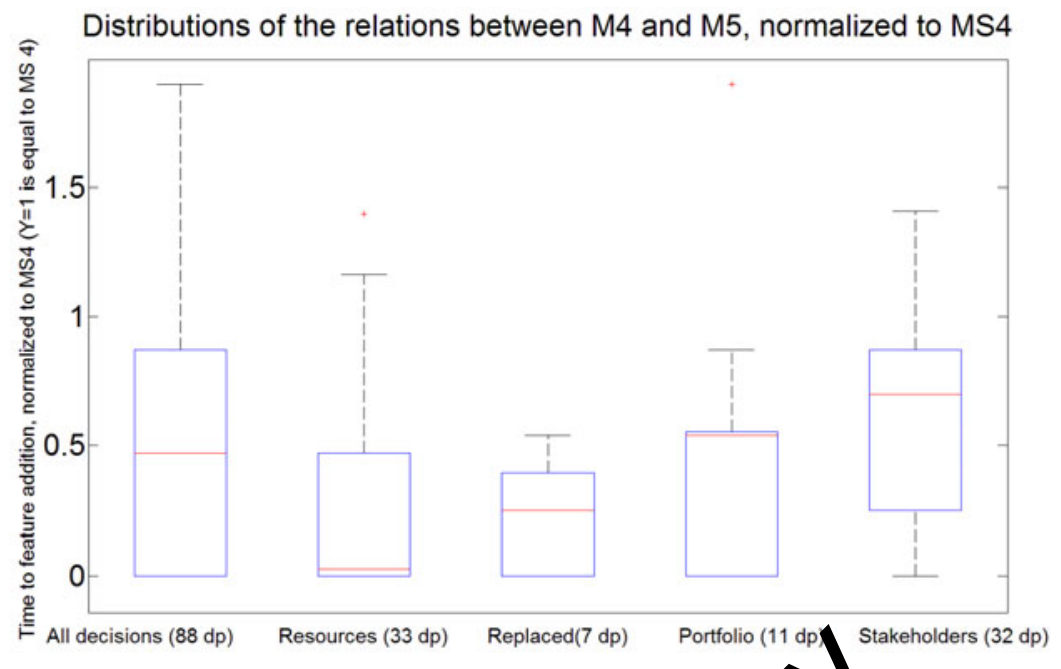

Fig. 3. The Correlation between the Time to feature ad and gn The Reason for Cancellation, normalized to MS4 of the project

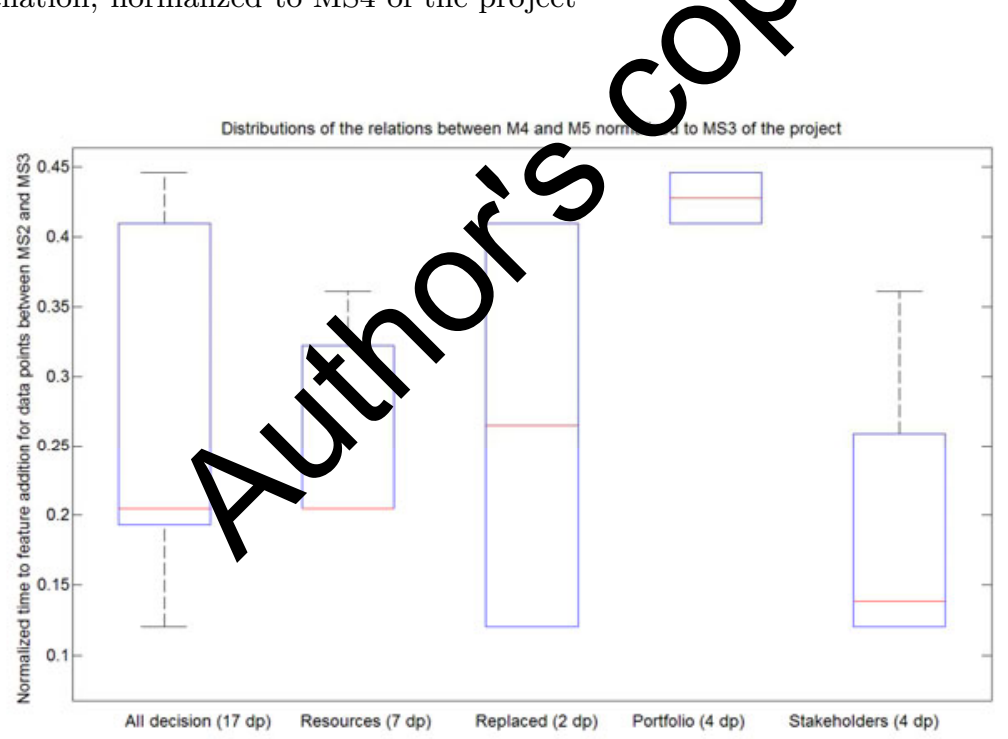

Fig. 4. The Correlation between the Time to feature addition and The Reason for Cancellation, normalized to MS3 of the project

values. However, we are cautious of drawing strong conclusions due to the relatively small data set. There is some indication of a relatively binary decision making process: cancel due to lack of resources or defer to a later date (portfolio changes + stakeholder business decision). We present this visualization to draw attention to the challenges faced with analyzing the tails of distributions in support of management decisions. 


\section{Threats to Validity}

In this section, threats to validity are outlined and discussed based on the classification by Wohlin et al [28]. The causal conclusions drawn from our analyses were validated in a number of meetings with practitioners who confirmed that frequent (and sometimes late) scope changes are principally caused by specific market forces. However, more research is needed to validate the causal influence of the different reasons for adding and removing features from the scope of the project. The causal influence for the timing of when the features were included or excluded from the scope of the analyzed projects also requires further investigation.

The proposed visualization do not promote particular reasons for including or excluding features from the scope of the project. Therefore, researcher bias toward (or fishing for) a specific justification is minimized. Finally, although the example visualizations show four main reasons for removing features from the scope of the project, there is no theoretical limit to the number of attributes that can be visualized using this technique. As a result, the o-operation threat for under-representation of the construct is minimize

With respect to external validity threats, we wa to emphasize that while the investigated scenario has been observe in specific company, using a specific development paradigm and releasin sctware products to a specific market, the identified issues of estimation chalrnges and changing the scope of the project are known and reported entware engineering literature. We are aware that the approach should o vated in one or more independent contexts, yet we believe that the puned visualizations have sufficient support in the current context that they can be applied, with appropriate caution, in other than studied contexts.

\section{Conclusions and Future Work}

Software engineerin as an engineering discipline, should be guided by the goal of value creation, me sured in terms that count for the enterprise that is investing the resources $[9$. In order for software systems to best catalyze their potential for novel value generation, management must maintain a broad perspective to ensure that investments is software artifacts deliver an acceptable return. In this context, deciding which requirements to include into the scope of an upcoming project is crucial for the process of value creation. In a rapidly changing situation, such as MDRE, the failure to quickly cancel features or projects that new information shows are unlikely to succeed is a common example of failing to make a value-optimizing decision [9. Improving the understanding of the connections between technical decisions and enterprise-level value maximization will enable software engineers and managers to make better choices.

In this paper, we present visualizations that emphasize the relationship between the technical and financial aspects of scoping decisions. Utilizing a particular industrial example, with rapidly changing context and a high degree of 
uncertainty, we demonstrate methods for analyzing the impact of scoping decision on the financial aspects of the project and the company (RQ1). The visualizations help to understand the drivers of late scope exclusions and inclusions and can assist management efforts to control them (RQ2). The results from this paper support both diagnostic and predictive aspects of decision making [29] and have been expressed in a manner that supports strategic decision management for the project.

In future work, we hope to obtain other data sets that will allow us to further generalize this work. We are particularly interested in investigating whether new reasons for removing features from the scope of the project, other than those identified in this work, can be identified as influences on the scoping process. A larger dataset may enable us to propose a method for minimizing waste incurred by late scope removal; we are particularly interested in temporal optimizations based on cost-benefit analysis or return on investment. Formal statistical analyses of the current dataset are in progress; additional datasets are expected to strengthen the results of these analyses. Finally, the investig tion of possible similarities between the defect detection and correction promand the associated costs are considered within the future work agenda.

In the visualization domain, we plan to investig feedback using geometry (such as line thickness proportional to cost) and luminance (proportional to cost). Cost feedback can also be provided u ing cost as a function of assessed (predicted) risk. Finally, further empirical studies are planned that investigate the utility of the visualizations for oct managers and how the visualizations are interpreted by them.

\section{Acknowledgments}

This work is supported by NOVA (Swedish Agency for Innovation Systems) within the UPITER pros

\section{References}

1. Regnell, B., Brinkkemper, S.: Market-Driven Requirements Engineering for Software Products. In: Engineering and Managing Software Requirements, pp. 287-308. Springer, Heidelberg (2005)

2. Chen, J., Reilly, R.R., Lynn, G.S.: The impacts of speed-to-market on new product success: the moderating effects of uncertainty. IEEE Transactions on Engineering Management 52(2), 199-212 (2005)

3. McPhee, C., Eberlein, A.: Requirements engineering for time-to-market projects. In: Proc. Ninth Annual IEEE Int. Conf. and Workshop on the Eng. of ComputerBased Systems, pp. 17-24 (2002)

4. Schumpeter, J.: Capitalism, Socialism and Democracy. Harper (1942)

5. Karlsson, J., Ryan, K.: A cost-value approach for prioritizing requirements. IEEE Software 14(5), 67-74 (1997)

6. Schmid, K.: A comprehensive product line scoping approach and its validation. In: 24th Int. Conf. on Soft. Eng (ICSE 2002), pp. 593-603 (2002) 
7. Regnell, B., Beremark, P., Eklundh, O.: A market-driven requirements engineering process - results from an industrial process improvement programme. Requirements Engineering Journal 3(2), 121-129 (1998)

8. Institute, P.M.: A Guide To The Project Management Body of Knowledge, 4th edn. Project Management Institute (2009)

9. Boehm, B., Sullivan, K.: Software economics: a roadmap. In: Proc. of the Conf. on The Future of Soft. Eng., ICSE 2000, Limerick, Ireland, pp. 319 343. ACM, New York (2000), http://doi.acm.org/10.1145/336512.336584, doi: $10.1145 / 336512.336584$

10. Wnuk, K., Regnell, B., Karlsson, L.: Visualization of feature survival in platformbased embedded systems development for improved understanding of scope dynamics. In: Third Int. Workshop on Req. Eng. Visualization (REV 2008), pp. 41-50 (2008)

11. Wnuk, K., Regnell, B., Karlsson, L.: Feature transition charts for visualization of cross-project scope evolution in large-scale requirements engineering for product lines. In: Forth Int. Workshop on Req. Eng. Visualization (REV 2009), pp. 89-98 (2009)

12. Wnuk, K., Regnell, B., Karlsson, L.: What happened to our Natures? visualization and understanding of scope change dynamics in a large dustrial setting. In: Proc. of the 17th IEEE Int. Req. Eng. Conference (R 20 9),pp. 89-98 (2009)

13. Robson, C.: Real World Research. Blackwell Pub sh, Malden (2002)

14. van de Weerd, I., Brinkkemper, S., Nieuwen Versendaal, J.A.: A reference framework for software product managen ent Technical Report UU-CS, vol. 2006(014), Utrecht (2006)

15. Karlsson, J.: A Systematic Approach Goritizing Software Requirements. Doctorial Dissertation, PhD thesis, Lin oping niversity, Sweden (1998)

16. Jorgensen, M., Shepperd, M.: A sotic review of software development cost estimation studies. IEEE Transa tion s on Software Engineering 33(1), 33-53 (1992)

17. Svahnberg, M., Gorschek,, ar, R., Torkar, R., Saleem, S.B., Shafique, M.U.: A systematic review on tegic release planning models. Inf. Softw. Technol. 52, 237-248 (2010 tp://dx.doi.org/10.1016/j.infsof.2009.11.006 doi: $10.1016 /$ j.infarf.20 9.1 7.006

18. Gorschek, T., G me 1., Pettersson, A., Torkar, R.: Market-driven requirements engineering proce $\$$ maturity model. Journal of Software Maintenance tba (tba 2010) (2010)

19. Khurum, M., Gorschek, T.: A systematic review of domain analysis solutions for product lines. J. Syst. Softw. 82, 1982-2003 (2009), doi:10.1016/j.jss.2009.06.048

20. Carlshamre, P., Sandahl, K., Lindvall, M., Regnell, B., Nattoch Dag, J.: An industrial survey of requirements interdependencies in software product release planning. In: Proceedings of the Fifth IEEE Int. Symp. on Req. Eng., RE 2001, p. 84. IEEE Computer Society, Washington, DC, USA (2001)

21. Wiess, D.M., Lai, C.T.R.: Software Product Line Engineering. Addison-Wesley, Reading (1999)

22. Green, P.E., Krieger, A.M.: Models and heuristics for product line selection. Marketing Science 4(1), 1-19 (1985), http://www.jstor.org/stable/183706

23. Tufte, E.: Envisioning Information. Graphics Press LLC (1990)

24. Gotel, O.C.Z., Marchese, F.T., Morris, S.J.: On requirements visualization. In: Proc. of the Second Int. Workshop on Req. Eng. Visualization (REV 2007), pp. 80-89 (2007) 
25. Pohl, K., Bockle, G., van der Linden, F.J.: Software Product Line Engineering: Foundations, Principles and Techniques. Springer, Heidelberg (2005)

26. Cooper, R.G.: Stage-gate systems: A new tool for managing new products. Business Horizons 33(3), 44-54 (1990)

27. Boehm, B., Basili, V.: Software defect reduction top 10 list. IEEE Computer 34(1), 135-137 (2001)

28. Wohlin, C., Runeson, P., Höst, M., Ohlsson, M.C., Regnell, B., Wesslen, A.: Experimentation in Software Engineering An Introduction. Kluwer Academic Publishers, Dordrecht (2000)

29. Pomerol, J.C.: Scenario Development and Practical Decision Making under Uncertainty: Application to Requirements Engineering. Requirements Engineering 3, 3-4 (1998)

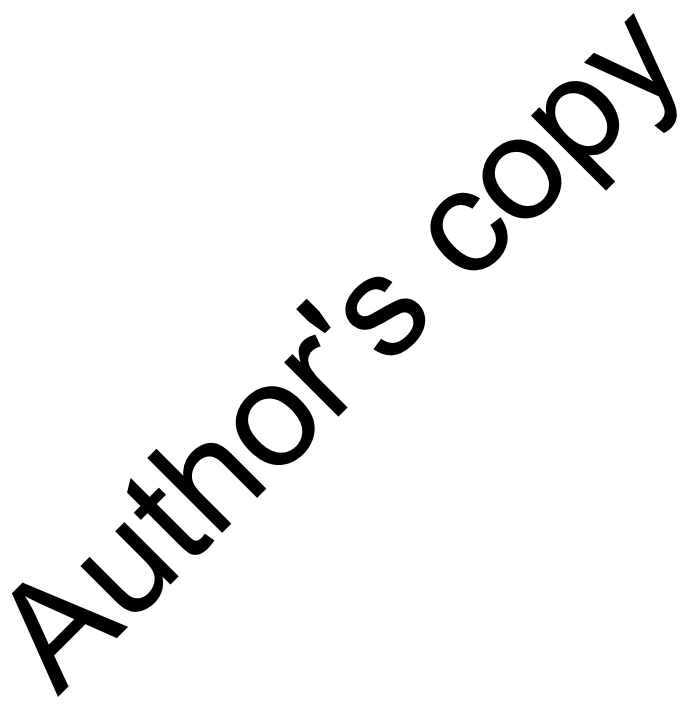

